Document downloaded from:

http://hdl.handle.net/10251/48887

This paper must be cited as:

Sánchez Pérez, EA.; Dirk (2011). The p-Daugavet property for function spaces. Archiv der Mathematik. 96(6):565-575.

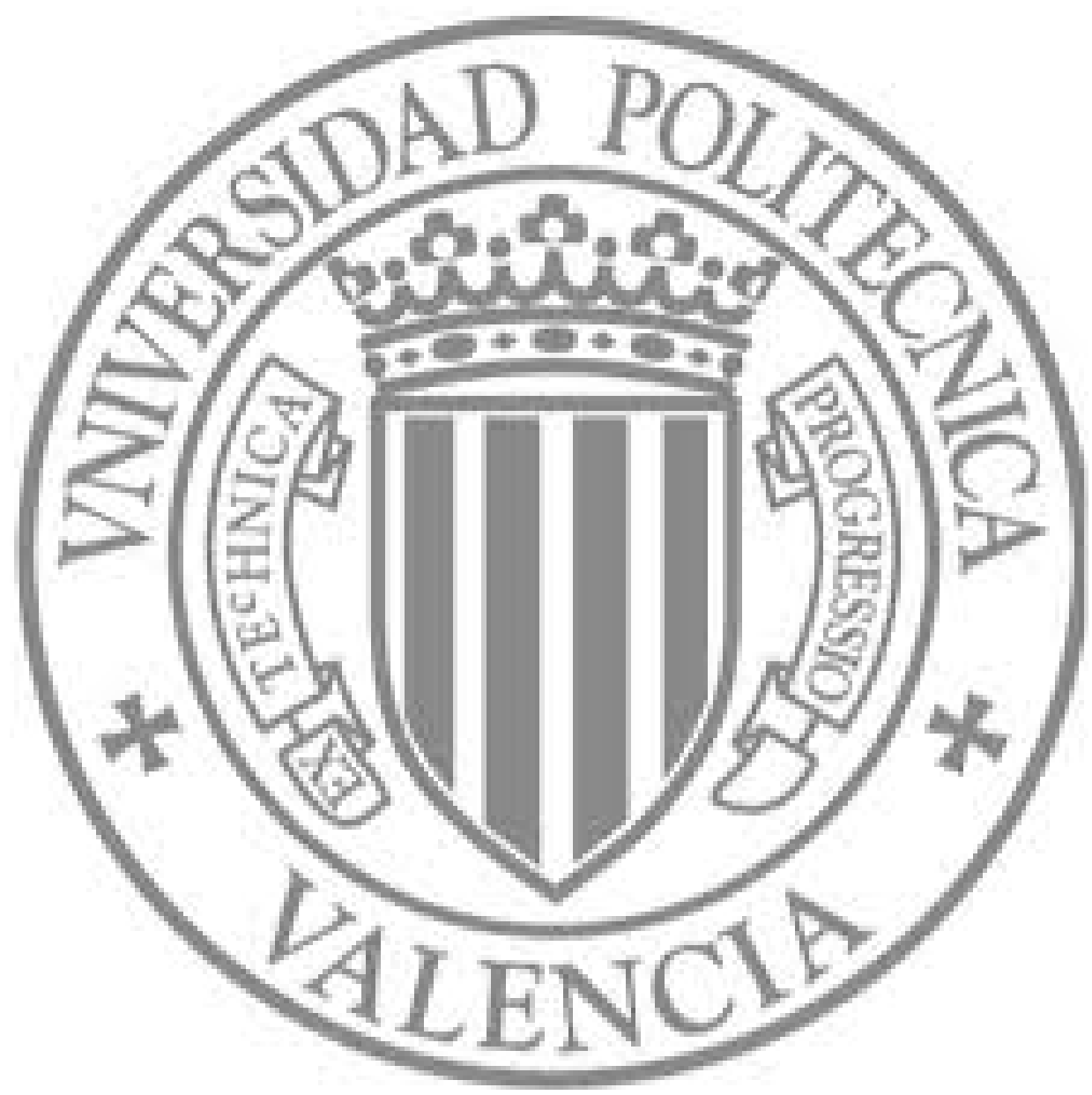

The final publication is available at

http://dx.doi.org/10.1007/s00013-011-0257-y

Copyright Springer Verlag (Germany) 


\title{
THE $p$-DAUGAVET PROPERTY FOR FUNCTION SPACES
}

\author{
ENRIQUE A. SÁNCHEZ PÉREZ AND DIRK WERNER
}

\begin{abstract}
A natural extension of the Daugavet property for $p$-convex Banach function spaces and related classes is analysed. As an application, we extend the arguments given in the setting of the Daugavet property to show that no reflexive space falls into this class.
\end{abstract}

\section{INTRODUCTION}

A Banach space $B$ is said to have the Daugavet property if every rank one operator $T: B \rightarrow B$ satisfies the Daugavet equation

$$
\|\mathrm{Id}+T\|=1+\|T\|,
$$

where Id is the identity map on $B$. In the setting of Banach function spaces, several recent papers have analysed for which ones the Daugavet property is satisfied. The main examples are $L^{1}(\mu)$ and $L^{\infty}(\mu)$ whenever $\mu$ does not have any atoms (see [7, Section 2]). Also, if $K$ is a compact Hausdorff topological space without isolated points, the space $C(K)$ has the Daugavet property; this space is a function space, but not a Banach function space in the sense that we use in this paper.

Some modifications of the Daugavet property have also been introduced in order to provide a weaker property for extending the techniques and results that arises for spaces with the Daugavet property to a bigger class of spaces (see for example [4, 9, 10, 11]). In this paper we consider what we call generalized function spaces (to be defined at the end of the introduction), a new class that includes $p$-convex Banach function spaces and $C(K)$-spaces, whose definition is partially motivated by the one of a $K$-representable space given in [5]. Our generalization of the Daugavet property is the following.

Definition 1.1. A constant $1 p$-convex (generalized) function space $X$ is said to have the $p$-Daugavet property if and only if for every rank one operator $T: X \rightarrow X$, the equation

$$
\sup _{f \in B_{X}}\left\|\left(f^{p}+T(f)^{p}\right)^{1 / p}\right\|=\left(1+\|T\|^{p}\right)^{1 / p}
$$

is fulfilled. We call this equation for an operator $T$ the $p$-Daugavet equation.

We remark that we deal with a different $p$-version of the Daugavet property in our paper [13].

2000 Mathematics Subject Classification. Primary 46B04; secondary 46B25.

Key words and phrases. Daugavet property, $L_{p}$-space.

The first author was partially supported by a grant from the Generalitat Valenciana (BEST/2009/108) and a grant from the Universidad Politécnica de Valencia (PAID-0009/2291). Support of the Ministerio de Educación y Ciencia, under project \#MTM200914483-C02-02 (Spain) and FEDER is also gratefully acknowledged. 
The aim of this paper is to characterize the $p$-Daugavet property and provide a description of the structure of the spaces that satisfy it, as well as to exhibit a class of spaces having the property. As applications, and following the same steps as for spaces with the Daugavet property, we prove that no reflexive constant $1 p$-convex generalized function space has the $p$ Daugavet property. From the methodological point of view, we adopt the Banach lattice aproach to the Daugavet property (see [1, 2]) but use the geometric tools that were introduced later ([7]).

We use standard notation. All the Banach spaces considered in the paper are supposed to be defined over the reals. Let $Y$ be a Banach space. $B_{Y}$ and $S_{Y}$ are the (closed) unit ball and the unit sphere, respectively. $Y^{*}$ denotes the dual space of $Y$. The slice $S\left(y^{*}, \varepsilon\right)$ defined by $y^{*} \in B_{Y^{*}}$ and $\varepsilon>0$ is given by

$$
S\left(y^{*}, \varepsilon\right)=\left\{y \in B_{Y}:\left\langle y, y^{*}\right\rangle \geq 1-\varepsilon\right\} .
$$

Let $(\Omega, \Sigma, \mu)$ be a measure space. A Banach function space $X(\mu)$ is an ideal of the space $L^{0}(\mu)$ of classes of measurable functions (the usual $\mu$-a.e. order is considered) that is a Banach space with a lattice norm $\|\cdot\|$ such that for every $A \in \Sigma$ of finite measure, $\chi_{A} \in X(\mu)$ (see [8, Def. 1.b.17]). If the measure $\mu$ is fixed in the context we simply write $X$ for $X(\mu) . X(\mu)^{+}$ stands for the positive cone of $X(\mu)$ and in general, if $S \subset X(\mu)$, we will write $S^{+}$for its positive part $S \cap X(\mu)^{+}$.

Let us now recall two basic geometric properties of Banach lattices. If $X$ is a Banach lattice, it is called $p$-convex if there is a constant $K$ such that for each finite sequence $\left(x_{i}\right)_{i=1}^{n}$ in $X$,

$$
\left\|\left(\sum_{i=1}^{n}\left|x_{i}\right|^{p}\right)^{1 / p}\right\|_{X} \leq K\left(\sum_{i=1}^{n}\left\|x_{i}\right\|_{X}^{p}\right)^{1 / p} .
$$

An operator $T: X \rightarrow F$ on a Banach lattice $X$ is $p$-concave if there is a constant $k$ such that for every sequence $\left(x_{i}\right)_{i=1}^{n}$ in $X$,

$$
\left(\sum_{i=1}^{n}\left\|T\left(x_{i}\right)\right\|_{F}^{p}\right)^{1 / p} \leq k\left\|\left(\sum_{i=1}^{n}\left|x_{i}\right|^{p}\right)^{1 / p}\right\|_{X} .
$$

The quantities $M^{(p)}(X)$ and $M_{(p)}(T)$ are respectively the best constants in the above inequalities. If $T$ is the identity map, we also say that $X$ is $p$ concave and denote the corresponding constant by $M_{(p)}(X)$. Throughout the paper we assume that the $p$-convexity constants of the spaces are equal to 1 ; we will write that the space $X$ is constant $1 p$-convex for short.

In order to extend these lattice notions to a bigger family of spaces, we introduce the class of generalized function spaces (g.f.s. for short). Let $1 \leq p<\infty$. Let $\Delta$ be a set and consider a family of measure spaces $\left\{\left(A_{\delta}, \Sigma_{\delta}, \mu_{\delta}\right): \delta \in \Delta\right\}$. Let $\left(\mathbb{R}^{A_{\delta}}\right)_{\mu_{\delta}}$ be the space of classes of $\mu_{\delta}$-a.e. equal measurable functions, usually denoted by $L^{0}\left(A_{\delta}, \mu_{\delta}\right)$. For each element $f \in \prod_{\delta \in \Delta}\left(\mathbb{R}^{A_{\delta}}\right)_{\mu_{\delta}}$, the modulus $|f|$ is defined pointwise by $|f|(\delta)=|f(\delta)|$. Let $X$ be a Banach space of (classes of) functions $f \in \prod_{\delta \in \Delta}\left(\mathbb{R}^{A_{\delta}}\right)_{\mu_{\delta}}$ such that $|f| \in X$ and with a norm with lattice properties when the natural order inherited in $X$ from the product is considered. Thus, $X$ is a Banach space of functions whose values at each point $\delta$ are classes of $\mu_{\delta}$-a.e. equal real functions such that the modulus of each element belongs to the space, too, 
and with a lattice norm; notice that the counting measure on $A_{\delta}$ is also admissible, which is important for including $C(K)$ spaces. The measure $\mu_{\delta}$ will not be written explicitly if it is not relevant in the context.

Note that, if $f \in X$, we can write it at each point $\delta \in \Delta$ as $f(\delta)=$ $\operatorname{sign}\{f(\delta)\}|f(\delta)|$ and define the map $i_{p}$ from $X$ taking values in $\prod_{\delta \in \Delta}\left(\mathbb{R}^{A_{\delta}}\right)_{\mu_{\delta}}$ by means of its pointwise evaluation

$$
i_{p}(f)(\delta):=\operatorname{sign}\{f(\delta)\}|f(\delta)|^{p}, \quad f \in X .
$$

Here, $\operatorname{sign}\{a\}$ denotes the sign of the real number $a$. We use the notation

$$
f^{p}:=i_{p}(f)
$$

we caution the reader that for even integers $f^{p}$ need not be the same as $|f|^{p}$. The map $i_{p}$ is clearly an injection; we denote by $i_{1 / p}$ (i.e., $i_{1 / p}(g)=g^{1 / p}$ for every $\left.g \in i_{p}(X)\right)$, the inverse map.

We say that a Banach space of functions as above is a constant $1 p$-convex generalized function space if for every finite family of elements $x_{1}, \ldots, x_{n} \in$ $X$ the function $\left(\sum_{i=1}^{n} x_{i}^{p}\right)^{1 / p}$ belongs to $X$ and

$$
\left\|\left(\sum_{i=1}^{n}\left|x_{i}\right|^{p}\right)^{1 / p}\right\| \leq\left(\sum_{i=1}^{n}\left\|x_{i}\right\|^{p}\right)^{1 / p} .
$$

It can easily be seen that this class includes for instance constant 1 $p$-convex Banach function spaces (take $\Delta$ a singleton), $C(K)$-spaces and $C(K, Y)$-spaces, where $Y$ is a constant $1 p$-convex Banach function space.

\section{The $p$-Convexification of the Daugavet property}

Let $X$ be a constant $1 p$-convex g.f.s. Consider the linear space $F_{X, p}$ of functions from $X$ to the corresponding product $\prod_{\delta \in \Delta}\left(\mathbb{R}^{A_{\delta}}\right)_{\mu_{\delta}}$ that are finite sums of the elements of the set

$S_{X, p}=\left\{\phi: X \rightarrow \prod_{\delta \in \Delta}\left(\mathbb{R}^{A_{\delta}}\right)_{\mu_{\delta}}: \phi=i_{p} \circ T, T: X \rightarrow X\right.$ linear and continuous $\}$.

Define the function norm on the space $F_{X, p}$ by

$$
\|\psi\|_{F_{X, p}}:=\sup _{f \in B_{X}}\left\|\left(\sum_{i=1}^{n} \phi_{i}(f)\right)^{1 / p}\right\|_{X}^{p}, \quad \psi=\sum_{i=1}^{n} \phi_{i}(f) \in F_{X, p} .
$$

Clearly, this formula is independent of the representation that is used for the element $\phi$ as a sum of elements of $S_{X, p}$. It can easily be checked that it is a norm on the space $F_{X, p}$, just taking into account that $\left\||\cdot|^{1 / p}\right\|_{X}^{p}$ is a norm in $i_{p}(X)$ since $X$ is a constant $1 p$-convex g.f.s. and that the map $i_{p}$ is one-to-one.

Lemma 2.1. Let $X$ be a constant 1 p-convex g.f.s. Suppose that $T$ and $S$ are operators from $X$ into $X$ such that

$$
\sup _{f \in B_{X}}\left\|T(f)^{p}+\left.S(f)^{p}\right|^{1 / p}\right\|=\left(\|T\|^{p}+\|S\|^{p}\right)^{1 / p} .
$$

Then for every $\alpha, \beta \geq 0$,

$$
\sup _{f \in B_{X}}\left\|\left|(\alpha T(f))^{p}+(\beta S(f))^{p}\right|^{1 / p}\right\|=\left(\|\alpha T\|^{p}+\|\beta S\|^{p}\right)^{1 / p} .
$$


Proof. Consider the functions $\phi$ and $\varphi$ in $S_{X, p}$ defined by $\phi=i_{p} \circ T$ and $\varphi=i_{p} \circ S$ and note that $\|\phi\|_{F_{X, p}}=\|T\|^{p}$ and $\|\varphi\|_{F_{X, p}}=\|S\|^{p}$. Therefore, by (2.1) we have that

$$
\|\phi+\varphi\|_{F_{X, p}}=\|\phi\|_{F_{X, p}}+\|\varphi\|_{F_{X, p}} .
$$

This implies, by Lemma 11.4 in [2] (or [15, p. 78]), that for every couple of non-negative real numbers $a$ and $b$,

$$
\|a \phi+b \varphi\|_{F_{X, p}}=a\|\phi\|_{F_{X, p}}+b\|\varphi\|_{F_{X, p}} .
$$

But this can be rewritten as

$$
\sup _{f \in B_{X}}\left\|\left|a(T(f))^{p}+b(S(f))^{p}\right|^{1 / p}\right\|_{X}^{p}=a\|T\|^{p}+b\|S\|^{p} .
$$

Thus, the result holds just by considering $\alpha=a^{1 / p}$ and $\beta=b^{1 / p}$.

Proposition 2.2. Let $X$ be a constant 1 p-convex g.f.s. Suppose that T: $X \rightarrow X$ is the rank one operator given by $T(f):=\left\langle f, g^{*}\right\rangle g$, where $g^{*} \in X^{*}$ and $g \in X$. The following statements are equivalent:

(1) $\sup _{f \in B_{X}}\left\|\left|f^{p}+T(f)^{p}\right|^{1 / p}\right\|_{X}=\left(1+\|T\|^{p}\right)^{1 / p}$.

(2) For every $\varepsilon>0$ there is an element $h \in S\left(\frac{g^{*}}{\left\|g^{*}\right\|}, \varepsilon\right)$ such that

$$
\left\|\left(\frac{g}{\|g\|}\right)^{p}+\left.h^{p}\right|^{1 / p}\right\|^{p} \geq 2-2 \varepsilon .
$$

Proof. Let us prove first that (1) implies (2). By Lemma 2.1, we can assume that the norm of $T$ is one, just by replacing $T$ by $T /\|T\|=T /\|g\|\left\|g^{*}\right\|$, and representing the resulting operator with two norm one elements that we still denote by $g$ and $g^{*}$. Let $\varepsilon>0$. Take $h \in S_{X}$ such that

$$
\left\|\left|h^{p}+T(h)^{p}\right|^{1 / p}\right\|_{X}^{p} \geq 2-\varepsilon .
$$

We can assume that $\left\langle h, g^{*}\right\rangle \geq 0$ (otherwise, replace $h$ by $-h$ ). Notice first that since $X$ is constant $1 p$-convex,

$$
1+\left\langle h, g^{*}\right\rangle^{p}\|g\|_{X}^{p}=\left\|\left|h^{p}\right|^{1 / p}\right\|_{X}^{p}+\left\|\left|T(h)^{p}\right|^{1 / p}\right\|_{X}^{p} \geq 2-\varepsilon,
$$

which implies that $\left\langle h, g^{*}\right\rangle \geq(1-\varepsilon)^{1 / p} \geq 1-\varepsilon$. Consequently, $g \in S\left(g^{*}, \varepsilon\right)$. On the other hand, also by the constant $1 p$-convexity of $X$,

$$
\begin{aligned}
2-\varepsilon & \leq\left\|\left|h^{p}+T(h)^{p}\right|^{1 / p}\right\|_{X}^{p} \\
& \leq\left\|\left|h^{p}+g^{p}\right|^{1 / p}\right\|_{X}^{p}+\left\|\left|T(h)^{p}-g^{p}\right|^{1 / p}\right\|_{X}^{p} \\
& =\left\|\left|h^{p}+g^{p}\right|^{1 / p}\right\|_{X}^{p}+\left\|\left|\left(1-\left\langle h, g^{*}\right\rangle^{p}\right) g^{p}\right|^{1 / p}\right\|^{p} \\
& \leq\left\|\left|h^{p}+g^{p}\right|^{1 / p}\right\|_{X}^{p}+\left(1-\left\langle h, g^{*}\right\rangle^{p}\right)\left\|\left|g^{p}\right|^{1 / p}\right\|^{p} \\
& \leq\left\|\left|h^{p}+g^{p}\right|^{1 / p}\right\|_{X}^{p}+\varepsilon .
\end{aligned}
$$

This gives the result.

For the converse, first notice that the inequality

$$
\sup _{f \in B_{X}}\left\|\left|f^{p}+T(f)^{p}\right|^{1 / p}\right\|_{X}^{p} \leq 1+\|T\|^{p}
$$


always holds, by the constant $1 p$-convexity of $X$. By Lemma 2.1, we can assume that $\|g\|=1$ and $\left\|g^{*}\right\|=1$, and then $\|T\|=1$. Let $\varepsilon>0$ and $h \in S\left(g^{*}, \varepsilon\right)$ such that

$$
\left\|\left|g^{p}+h^{p}\right|^{1 / p}\right\|^{p} \geq 2-2 \varepsilon .
$$

Then, again by the constant $1 p$-convexity of $X$,

$$
\begin{aligned}
2-2 \varepsilon \leq\left\|\left|g^{p}+h^{p}\right|^{1 / p}\right\|^{p} & =\left\|\left|g^{p}-T(h)^{p}+T(h)^{p}+h^{p}\right|^{1 / p}\right\|^{p} \\
& \leq\left\|\left|g^{p}-T(h)^{p}\right|^{1 / p}\right\|^{p}+\left\|\left|T(h)^{p}+h^{p}\right|^{1 / p}\right\|^{p} \\
& \leq\left(1-\left\langle h, g^{*}\right\rangle^{p}\right)+\left\|\left|T(h)^{p}+h^{p}\right|^{1 / p}\right\|^{p} \\
& \leq\left(1-(1-\varepsilon)^{p}\right)+\left\|\left|T(h)^{p}+h^{p}\right|^{1 / p}\right\|^{p} .
\end{aligned}
$$

Since this holds for every $\varepsilon>0$, we obtain the result.

Example 2.3. Let $X(\mu)$ be a constant $1 p$-convex Banach function space. Consider the set $P$ of positive rank one operators from $X(\mu) \rightarrow X(\mu)$, i.e.,

$$
P=\left\{T: X \rightarrow X: T=g^{*} \otimes g, g^{*} \in\left(X(\mu)^{*}\right)^{+}, g \in(X(\mu))^{+}\right\} .
$$

Proposition 2.2 gives directly the following result by taking into account that the supremum in (1) of Proposition 2.2 can be computed using just positive elements.

The following assertions are equivalent:

(1) For every positive rank-one operator $T \in P$,

$$
\sup _{f \in B_{X}}\left\|\left|f^{p}+T(f)^{p}\right|^{1 / p}\right\|_{X}=\left(1+\|T\|^{p}\right)^{1 / p} .
$$

(2) For every $\varepsilon>0$, every $g \in S_{X}^{+}$and every $g^{*} \in S_{X^{*}}^{+}$there is an element $h \in\left(S\left(g^{*}, \varepsilon\right)\right)^{+}$such that

$$
\left\|\left|g^{p}+h^{p}\right|^{1 / p}\right\|^{p} \geq 2-2 \varepsilon .
$$

For instance, $L^{p}$-spaces satisfy the statements above, since they are $p$ concave and $M_{(p)}\left(L^{p}\right)=1$. This includes the case of $\ell^{p}$; recall that the Daugavet property is not satisfied for $\ell^{1}$, so the property given by the equivalent assertions above, at least for the case $p=1$, is strictly weaker than the Daugavet property. We will show that this is also the case for $p>1$ but in a more dramatic sense, since $L^{p}(\mu)$ over an atomless measure $\mu$ does not satisfy the $p$-Daugavet property. The reader can find more information about what is called the positive Daugavet property in [3, Section 5].

Besides this example and taking into account the purpose of this paper, the main application of Proposition 2.2 is the geometric characterization of the $p$-Daugavet property that is given in the following result.

Corollary 2.4. Let $X$ be a constant 1 p-convex g.f.s. The following are equivalent:

(1) $X$ has the $p$-Daugavet property.

(2) For every $\varepsilon>0$, every $g \in S_{X}$ and every $g^{*} \in S_{X^{*}}$ there is an element $h \in S\left(g^{*}, \varepsilon\right)$ such that

$$
\left\|\left|g^{p}+h^{p}\right|^{1 / p}\right\|^{p} \geq 2-2 \varepsilon .
$$


We now define a new class of spaces that we call $(p, \mathcal{K})$-representable spaces. This definition generalizes in a sense the one given in [5, Definition 2.3].

Definition 2.5. Let $1 \leq p<\infty$ and let $I$ be an index set. Consider a family $\mathcal{K}=\left\{K_{i}: i \in I\right\}$ of (disjoint) compact Hausdorff spaces. Let $X$ be a Banach space. We say that $X$ is $(p, \mathcal{K})$-representable if there exists a family $\left(X_{k}\right)_{k \in \bigcup_{i \in I} K_{i}}$ of constant $1 p$-convex Banach function spaces or $C(K)$-spaces such that:

(i) Each $x \in X$ can be identified linearly with its coordinates in the product $\prod_{k \in \cup_{i \in I} K_{i}} X_{k}$, and if $i \in I$, the restriction of $x$ to the product $\prod_{k \in K_{i}} X_{k}$ belongs to $X$. Also, for every finite family $x_{1}, \ldots, x_{n} \in$ $X$, the element $\left(\sum_{\nu=1}^{n} x_{\nu}^{p}\right)^{1 / p}$ that is defined pointwise by means of its representation belongs to $X$.

(ii) Consider the space $\bigoplus_{i \in I}^{\infty} C\left(K_{i}\right)$, where the sup norm for the sum is considered. If $x \in X$, for every $\left(\varphi_{i}\right)_{i \in I} \in \bigoplus_{i \in I}^{\infty} C\left(K_{i}\right)$ the product $\left(\varphi_{i}\right) x=\left(\left.\varphi_{i} x\right|_{i}\right)$ belongs to $X$.

(iii) For every $x \in X$,

$$
\|x\|=\sup \left\{\left(\sum_{j \in F}\left\|x\left(k_{j}\right)\right\|^{p}\right)^{1 / p}: F \subset I \text { finite }, k_{j} \in K_{j}\right\}<\infty .
$$

(iv) For every $x \in X, i \in I$ and $\varepsilon>0$, the set

$$
\left\{k \in K_{i}:\|x(k)\| \geq(1-\varepsilon)\left\|\left.x\right|_{i}\right\|\right\}
$$

is infinite.

Remark 2.6.

(1) If $F$ is a finite subset of $I$, we write $\left.x\right|_{F}$ for the element $\left.x\right|_{F}=$ $\left.\sum_{i \in F} x\right|_{i}$ that coincides with the projection of $x$ in the coordinates belonging to $\prod_{k \in \bigcup_{i \in F} K_{i}} X_{k}$.

(2) Notice that since each space $X_{k}$ has lattice properties, by the description of the norm required in (iii) for every $\left(\varphi_{i}\right) \in B_{\bigoplus_{i \in I}^{\infty} C\left(K_{i}\right)}$ and $x \in X,\|\varphi x\| \leq\|x\|$.

(3) Straightforward calculations show that if the space $X$ is $(p, \mathcal{K})$ representable, then it is a constant $1 p$-convex g.f.s.

(4) The chief example of a $(p, \mathcal{K})$-representable space is the $\ell^{p}$-sum $\bigoplus_{i \in I}^{p} L^{\infty}\left(\mu_{i}\right)$; here $\mu_{i}$ is a positive nonatomic measure. Recall that every space $L^{\infty}(\Omega, \Sigma, \mu)$ is lattice isometrically isomorphic to some $C(K)$ with $K$ a compact Hausdorff space [14, p. 104], and if $\mu$ is nonatomic, then $K$ is perfect so that (iv) of Definition 2.5 holds. Note that, by construction, $\bigoplus_{i \in I}^{p} L^{\infty}\left(\mu_{i}\right)$ is a constant- $1 p$-convex g.f.s.

Proposition 2.7. If $X$ is $(p, \mathcal{K})$-representable, then it has the $p$-Daugavet property. 
Proof. Take two elements $x \in S_{X}$ and $x^{*} \in S_{X^{*}}$ and $\varepsilon>0$. There is a finite set $F_{1} \subset I$ such that

$$
(1-\varepsilon)^{1 / 2 p}\|x\| \leq\left(\sum_{i \in F_{1}}\left\|\left.x\right|_{i}\right\|^{p}\right)^{1 / p} .
$$

On the other hand, the set $\left\{\left.x\right|_{F}: x \in B_{X}, F \subset I\right.$ finite $\}$ is clearly dense in $B_{X}$ and hence norming for $X^{*}$. Consequently, there is a finite set $F_{2} \subset I$ and an element $z \in S_{X}$ such that

$$
\left(1-\frac{\varepsilon}{2}\right)\left\|x^{*}\right\| \leq\left\langle\left. z\right|_{F_{2}}, x^{*}\right\rangle .
$$

Take $F=F_{1} \cup F_{2}$ and $N=|F|$. As in the proof of Lemma 2.4 in [5], for each $i \in F$, (iv) in Definition 2.5 provides a sequence of different points $\left(w_{i, n}\right)_{n=1}^{\infty}$ in $K_{i}$ such that for each $n$,

$$
\left\|\left.x\right|_{i}\left(w_{i, n}\right)\right\|>(1-\varepsilon)^{1 / 2 p}\left\|\left.x\right|_{i}\right\| .
$$

An application of Uryson's lemma in each $K_{i}$ provides a sequence of normalised disjointly supported functions $\left(f_{i, n}\right)_{n=1}^{\infty}$ such that $f_{i, n}\left(w_{i, n}\right)=1$ for every $n$. For every $i \in F$, the sequence $\left(f_{i, n}\right)_{n}$ converges pointwise to 0 , and consequently it converges weakly to 0 in $C\left(K_{i}\right)$. Therefore, the function $\left(f_{i, n}\right): \prod_{i \in F} K_{i} \rightarrow \mathbb{R}^{N}$ defined in each coordinate as $f_{i, n}(w)$ converges weakly to 0 in $\bigoplus_{i \in F}^{\infty} C\left(K_{i}\right)$.

As a consequence of the requirements in Definition 2.5, for each $v \in X$, the linear map $J: \bigoplus_{i \in F}^{\infty} C\left(K_{i}\right) \rightarrow X$ given by $J\left(\left(\varphi_{i}\right)\right)=\left.\sum_{i \in F} \varphi_{i} v\right|_{i}$ is welldefined and continuous, and so weak-to-weak continuous. This implies that the sequence $\left(y_{n}\right)_{n=1}^{\infty}$, where

$$
y_{n}:=\left.\sum_{i \in F} f_{i, n} x\right|_{i}+\left.\sum_{i \in F_{2}}\left(1-f_{i, n}\right) z\right|_{i}
$$

(note that only $F_{2}$ appears in the second sum) converges weakly to $\left.z\right|_{F_{2}}$ in $X$. Notice that all these elements have norm $\leq 1$. Therefore there is an index $m$ such that $\left\langle y_{m}, x^{*}\right\rangle>1-\varepsilon$. Take $y:=y_{m}$. Finally, note that

$$
\begin{aligned}
\left\|\left|x^{p}+y^{p}\right|^{1 / p}\right\| & \geq\left(\sum_{i \in F}\left\||x|_{i}^{p}+\left.\left.y\right|_{i} ^{p}\right|^{1 / p}\right\|^{p}\right)^{1 / p} \\
& \geq\left(\sum_{i \in F}\left\|\left|x\left(w_{i, m}\right)^{p}+y\left(w_{i, m}\right)^{p}\right|^{1 / p}\right\|^{p}\right)^{1 / p} \\
& =2^{1 / p}\left(\sum_{i \in F}\left\|x\left(w_{i, m}\right)\right\|^{p}\right)^{1 / p} \\
& \geq 2^{1 / p}(1-\varepsilon)^{1 / 2 p}\left(\sum_{i \in F}\left\|\left.x\right|_{i}\right\|^{p}\right)^{1 / p} \\
& \geq 2^{1 / p}(1-\varepsilon)^{1 / p}
\end{aligned}
$$

This completes the proof.

Corollary 2.8. The space $\bigoplus_{i \in I}^{p} L^{\infty}\left(\mu_{i}\right)$ has the p-Daugavet property. 
Remark 2.9. A direct consequence of the definition of $(p, \mathcal{K})$-representable spaces is that every representable space in the sense of [5, Definition 2.3], which is represented on a compact set $K$ and over a product $\prod_{k \in K} X_{k}$ of constant $1 p$-convex Banach function spaces or $C(K)$-spaces and for which the requirements in (i) of Definition 2.5 are fulfilled, is in fact $(p, \mathcal{K})$ representable; it is enough to consider a one point set $I$.

\section{The $p$-Daugavet equation for Weakly compact operators}

The proofs of the following results hold by adapting the techniques used in the ones for the case of spaces with the Daugavet property, so we only sketch the parts that are different. For the Daugavet property, Theorem 3.1 was first proved in [7] and Proposition 3.4 in [6].

Theorem 3.1. Let $X$ be a constant 1 p-convex g.f.s. and with the $p$-Daugavet property. Then every weakly compact operator satisfies the $p$-Daugavet equation. Consequently, $X$ cannot be reflexive.

Proof. Let us show first the following claim:

- Let $X(\mu)$ be a constant 1 g.f.s. If for every $\varepsilon>0$ there are a slice $S\left(g^{*}, \delta\right)$ and an element $g \in S_{X}$ such that $T\left(S\left(g^{*}, \delta\right)\right)$ is included in the ball $B_{\varepsilon}(g)$, then $T$ satisfies the $p$-Daugavet equation.

In order to see this, note that we can assume that $\|T\|=1$. Take $\varepsilon>0$, and note that we can also assume that $0<\delta \leq \varepsilon$. By Proposition 2.2 there is an element $h \in S\left(g^{*}, \delta\right)$ such that $\left\|\left(h^{p}+g^{p}\right)^{1 / p}\right\|_{X} \geq 2-2 \varepsilon$; it follows that $\|T(h)-g\|_{X} \leq \varepsilon$. Thus

$$
\begin{aligned}
\sup _{f \in B_{X}}\left\|\left(f^{p}+T(f)^{p}\right)^{1 / p}\right\|_{X}^{p} & \geq\left\|\left(h^{p}+T(h)^{p}\right)^{1 / p}\right\|_{X}^{p} \\
& \geq\left\|\left(h^{p}+g^{p}-\left(g^{p}-T(h)^{p}\right)\right)^{1 / p}\right\|_{X}^{p} \\
& \geq\left\|\left(h^{p}+g^{p}\right)^{1 / p}\right\|_{X}^{p}-\left\|\left(g^{p}-T(h)^{p}\right)^{1 / p}\right\|_{X}^{p} \\
& \geq 2-2 \varepsilon-\left\|\left(g^{p}-T(h)^{p}\right)^{1 / p}\right\|_{X}^{p} .
\end{aligned}
$$

Now notice that the inequality

$$
\left\|\left|g^{p}-T(h)^{p}\right|^{1 / p}\right\|_{X}^{p} \leq\|g-T(h)\|^{p}+p(2 k(p))^{p / p^{\prime}}\|g-T(h)\|,
$$

holds (Lemma 2.4 in 13]), where $k(p)$ is defined to be 1 if $p \geq p^{\prime}$ and $k(p)=2^{\left(p^{\prime} / p\right)-1}$ if $p<p^{\prime}$; it can be proved using the pointwise estimates given in [12, Section 2.2], Hölder's inequality for Banach function spaces and the constant $1 p$-convexity of $X$. Therefore, we obtain that

$$
2-2 \varepsilon-\|g-T(h)\|_{X}^{p} \geq 2-2 \varepsilon-\varepsilon^{p}-p(2 k(p))^{p / p^{\prime}} \varepsilon .
$$

Since this holds for every $\varepsilon>0$, we have proved the claim.

For finishing the proof let $\varepsilon>0$ and take into account that we are assuming that the norm closure $\overline{T\left(B_{X}\right)}=K$ is a weakly compact set, and therefore it is the closed convex hull of its strongly exposed points. Thus, there is a strongly exposed point $f_{0} \in K$ such that $1-\varepsilon / 2<\left\|f_{0}\right\| \leq 1$. Then there is a slice $S$ such that $T(S) \subset B_{\varepsilon}\left(f_{0}\right)$ (see the proof of this for example in [2, Theorem 11.50] or [7]). Since $B_{\varepsilon / 2}\left(f_{0}\right) \subset B_{\varepsilon}\left(f_{0} /\left\|f_{0}\right\|\right)$, the claim gives the result. Finally, note that the space $X$ cannot be reflexive, for otherwise the operator -Id would satisfy the $p$-Daugavet equation. 
A relevant consequence of Theorem 3.1 is that no $L^{p}(\mu)$ space for $1<p<$ $\infty$ satisfies the $p$-Daugavet property; recall that for $p=1$ and an atomless measure $\mu$ the space $L^{1}(\mu)$ has the Daugavet property (see [1, Theorem 3.2] or [7, Example, p. 858]). Here is another corollary.

Corollary 3.2. Let $1<p<\infty$. If a constant $1 p$-convex Banach function space has the p-Daugavet property, then every p-concave operator satisfies the $p$-Daugavet equation.

Proof. Each such operator factorizes through an $L^{p}$ space (see [8, Corollary 1.d.12]), and so it is weakly compact.

Related arguments can also be used for proving that there are no ideals in such spaces being isomorphic to $L^{p}$ spaces.

Corollary 3.3. In a constant $1 p$-convex Banach function space with the $p$-Daugavet property, $p>1$, there are no $p$-concave band projections.

Proof. Assume that $Q: X \rightarrow X$ is such a projection; there is another disjoint projection $P$ such that $\mathrm{Id}=Q+P$. Then

$$
\begin{aligned}
\sup _{f \in B_{X}}\left\|\left(f^{p}-Q(f)^{p}\right)^{1 / p}\right\| & =\sup _{f \in B_{X}}\left\|\left((P(f)+Q(f))^{p}-Q(f)^{p}\right)^{1 / p}\right\| \\
& =\sup _{f \in B_{X}}\left\|\left(P(f)^{p}+Q(f)^{p}-Q(f)^{p}\right)^{1 / p}\right\| \\
& =\sup _{f \in B_{X}}\|P(f)\| \leq 1<\left(1+\|Q\|^{p}\right)^{1 / p}
\end{aligned}
$$

which contradicts Corollary 3.2 , since $-Q$ is $p$-concave.

Moreover, the same computation gives that in a constant $1 p$-convex Banach function space with the $p$-Daugavet property there are no projection bands being isomorphic to $L^{p}$ spaces or to any reflexive Banach space. Finally, note that if $X$ is order continuous, then every ideal is the range of a positive contractive projection, so there are no ideals isomorphic to $L^{p}$ spaces as Banach spaces (see [8, Proposition 1.a.11]).

Let us finish the paper with a suitable version of the non-existence of unconditional bases for Banach spaces with the $p$-Daugavet property. We recall that no Banach space with the Daugavet property has an unconditional basis 6 and does not even embed into a space with an unconditional basis [7]. Let $X$ be a g.f.s. and consider an unconditional basis $\mathbf{B}:=\left\{e_{n}\right.$ : $n \in \mathbb{N}\}$ with projections $P_{A}$, where $A$ is a finite subset of $\mathbb{N}$. Write $Q_{A}$ for the complementary projection $\mathrm{Id}-P_{A}$. We say that $\mathbf{B}$ satisfies a lower $p$-estimate if there is a constant $k>0$ such that

$$
\left\|Q_{A}\right\| \geq \sup _{f \in B_{X}}\left\|\left(k f^{p}-P_{A}(f)^{p}\right)^{1 / p}\right\| .
$$

Notice that every unconditional basis satisfies a lower 1-estimate with the constant $k=1$. (Indeed, this is so for every Schauder basis.) Also, if the natural lattice structure associated to an unconditional basis is considered and $X$ becomes a g.f.s. over the counting measure, the basis satisfies a lower $p$-estimate for every $1 \leq p<\infty$, also with the constant $k=1$. None of these cases can occur if $X$ is a Banach space with the Daugavet property or 
a constant $1 p$-convex g.f.s. with the $p$-Daugavet property, respectively. The following result generalizes these examples.

Proposition 3.4. Let $X$ be a constant 1 p-convex g.f.s. that has the $p$ Daugavet property. Then $X$ does not have an unconditional basis with a lower p-estimate.

Proof. Let $\mathbf{B}:=\left\{e_{n}: n \in \mathbb{N}\right\}$ be an unconditional basis for $X$ with a lower $p$-estimate. For every finite subset $A \subset \mathbb{N}$ denote by $P_{A}$ and $Q_{A}$ the corresponding projections on the subspaces generated by the elements of the basis with subscripts in $A$ and $\mathbb{N} \backslash A$, respectively. Since Id $=P_{A}+Q_{A}, \mathbf{B}$ has a lower $p$-estimate and by Theorem $3.1-P_{A}$ satisfies the $p$-Daugavet equation, there is a constant $k>0$ such that

$$
\left\|Q_{A}\right\| \geq \sup _{f \in B_{X}}\left\|\left|k f^{p}+\left(-P_{A}(f)\right)^{p}\right|^{1 / p}\right\|_{X}=\left(k^{1 / p}+\left\|P_{A}\right\|^{p}\right)^{1 / p} .
$$

If we define $V=\sup \left\{\left\|P_{A}\right\|: A\right.$ finite $\}$ and $W=\sup \left\{\left\|Q_{A}\right\|: A\right.$ finite $\}$, clearly $W \leq V$. Since by the inequalities above $W \geq\left(k^{1 / p}+V^{p}\right)^{1 / p}$, we obtain that $V=W=\infty$, a contradiction with the fact that $\mathbf{B}$ is an unconditional basis.

\section{REFERENCES}

[1] Yu.A. Abramovich, C.D. Aliprantis, and O. Burkinshaw. The Daugavet equation in uniformly convex Banach spaces. J. Funct. Anal. 97 (1991), 215-230.

[2] Yu.A. Abramovich and C.D. Aliprantis. An Invitation to Operator Theory. Graduate Studies in Mathematics, Vol. 50. Amer. Math. Soc., Providence RI, 2002.

[3] D. Bilik, V. Kadets, R. Shvidkoy, And D. Werner. Narrow operators and the Daugavet property for ultraproducts. Positivity 9 (2005), 45-62.

[4] K. Boyko And V. Kadets. Daugavet equation in $L_{1}$ as a limiting case of the Benyamini-Lin $L_{p}$ theorem. Kharkov National University Vestnik 645 (2004), 22-29.

[5] J. Becerra Guerrero and A. Rodríguez-Palacios. Banach spaces with the Daugavet property, and the centralizer. J. Funct. Anal. 254 (2008), 2294-2302.

[6] V.M. KADETS. Some remarks concerning the Daugavet equation. Quaestiones Math. 19 (1996), 225-235.

[7] V. Kadets, R. Shvidkoy, G. Sirotkin, And D. Werner. Banach spaces with the Daugavet property. Trans. Amer. Math. Soc. 352 (2000), 855-873.

[8] J. Lindenstrauss and L. Tzafriri. Classical Banach Spaces II. Springer, BerlinHeidelberg-New York, 1979.

[9] M. Martín. The Daugavetian index of a Banach space. Taiwan. J. Math. 7 (2003), 631-640.

[10] M. Martín And T. OikhBerG. An alternative Daugavet property. J. Math Anal. Appl. 294 (2004), 158-180.

[11] T. OikHBerg. Spaces of operators, the $\psi$-Daugavet property, and numerical indices. Positivity 9 (2005), 607-623.

[12] S. OkAdA, W.J. Ricker, And E.A. SÁnchez PÉRez. Optimal Domain and Integral Extension of Operators acting in Function Spaces. Operator Theory: Adv. Appl., vol. 180. Birkhäuser, Basel, 2008.

[13] E.A. SÁnchez PÉRez And D. Werner. The geometry of $L^{p}$-spaces over atomless measure spaces and the Daugavet property. Banach J. Math. Anal. 5, No. 1 (2011), $167-180$

[14] H.H. Schaefer. Banach Lattices and Positive Operators. Springer, BerlinHeidelberg-New York, 1974.

[15] D. Werner. Recent progress on the Daugavet property. Irish Math. Soc. Bulletin 46 (2001), 77-97. 
Instituto Universitario de Matemática Pura y Aplicada, Universidad Politécnica de Valencia, Camino de Vera s/n, 46071 Valencia, Spain.

E-mail address: easancpe@mat.upv.es

Department of Mathematics, Freie Universität Berlin, Arnimallee 6, D-14 195 Berlin, Germany

E-mail address: werner@math.fu-berlin.de 